\title{
The lethality of sex
}

Drosophila melanogaster controls somatic sex determination via the master-switch Sex-lethal (Sxl). In somatic cells, Sxl expression is 'off' in male somatic cells and 'on' in females, where it regulates splicing and translation. Female flies also use Sxl during oogenesis to control mitosis and meiotic recombination in the germ cells, in which Sxl dramatically changes localization from the cytoplasm to the nucleus during germ-cell maturation. New work by Cynthia Vied and Jamila Horabin (Development 128, 2649-2660; 2001) indicates that the Hedgehog $(\mathrm{Hh})$ signalling pathway controls some of these changes in Sxl localization within the female germ line.

Hh controls the development of many different tissues and signals through its receptor Patched (Ptc). When Hh is bound to its receptor it relieves the repression normally placed by Ptc on Smoothened (Smo). Once this repression is relieved, Smo activates the transcription factor Cubitus interruptus (Ci) through a downstream signalling pathway that includes Fused $(\mathrm{Fu})$ and Costal-2 (Cos-2). In the presence of Hh signalling, $\mathrm{Ci}$ is processed into an activated isoform with a relative molecular mass of $155,000\left(M_{\mathrm{r}} 155 \mathrm{~K}\right)$, which moves into the nucleus to affect downstream gene expression. Without $\mathrm{Hh}$, it is proposed that $\mathrm{Ci}$ is tethered to cytoplasmic microtubules as a part of a complex with Cos-2, Fu, $\beta$-tubulin and $\gamma$-tubulin, which leads to its processing into repressor form of $M_{\mathrm{r}} 75 \mathrm{~K}$.

Vied and Horabin showed that the intracellular localization of Sxl in early female germ cells is affected by the Hh pathway. When the Hh pathway was activated, the authors saw

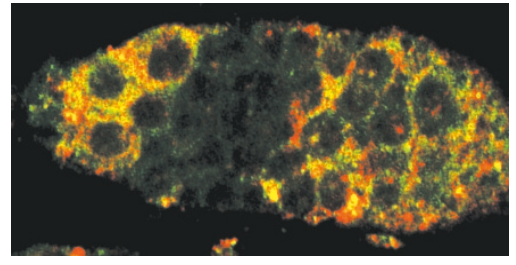

CYNTHIA VIED AND JAMILA HORABIN

changes in the localization of Ptc and Fu, which were similar to those of Sxl (picture shows co-localization of Sxl and Fu in the germarium (yellow)). In addition, Sxl was found in a complex with $\mathrm{Fu}, \mathrm{Cos}-2$ and $\gamma$-tubulin. This led the authors to propose that the whole complex, including Sxl, is found on microtubules in the absence of $\mathrm{Hh}$ signalling. During signalling, the complex is released from the microtubules and moves to the nucleus to elicit the required downstream responses. It also seems that the Hh pathway affects Sxl protein levels within these early germ cells, possibly through degradation.

As this is the first target of the Hh pathway that is not $\mathrm{Ci}$, the implications of this work are far-reaching. Drosophila geneticists must now determine whether the Hh pathway, or other signalling pathways including the Wingless pathway, work through different downstream effectors.

SARAH GREAVES define an upper limit to the likely separase targets in budding yeast.

A further implication of the Sullivan et al. paper is that separase-mediated cleavage might in some cases inactivate substrates (Scc1 and Rec8) but in other cases activate or modify them (Slk19). These different effects of separase action come, at least in part, from the nature of the newly exposed amino (N)-terminal residue ${ }^{12}$. Cleavage of Sccl by separase exposes an N-terminal arginine residue that directs rapid degradation of the cleaved Sccl fragment by the Nend rule $^{14}$. This is a satisfying result because, for a long time, the physiological implications of $\mathrm{N}$-end rule proteolysis were not known. In contrast to Scc1, separase cleavage of Slk19 exposes an N-terminal serine that, by the $\mathrm{N}$-end rule, results in a stable cleaved product (Fig. 1). Thus, the Nend rule provides the potential for enormous diversity in regulation of protein function, especially when one considers that there might be additional unidentified intracellular proteases. Although there were clear links between separase and proteolysis of Scc1 (ref. 6), it was initially not obvious from the Esp1 (encoding separase) sequence that it was, in fact, a protease. It took the bioinformatic expertise of Eugene
Koonin and colleagues to identify the caspase-like motifs and to pinpoint the active site $^{3}$.

That separase is important for mitotic spindle function does not come as a complete surprise. In fact, a role for Esp1 in spindle elongation in Schizosaccharomyces pombe $^{15}$ and Saccharomyces cerevisiae ${ }^{16}$ was suggested well before separase's role in dissolving sister cohesion was known. Separase was shown to localize to anaphase spindles in $S$. pombe $e^{17}$ and $S$. cerevisiae ${ }^{6,18}$, supporting a role in spindle function. However, it remained possible that the spindle defects in separase-deficient cells were a secondary consequence of the defect in separating sisters. A clear requirement for separase in spindle elongation was recently demonstrated in cells that were engineered to dissolve cohesion independent of separase activity. Even when sister cohesion was destroyed artificially, cells lacking separase activity still did not normally elongate their spindles $^{3,18}$. Sullivan et al. initiated the search for alternative separase targets with these observations in mind.

Is the anaphase spindle-elongation defect of separase-deficient cells caused by their failure to cleave Slk19? This pleasingly simple explanation cannot be correct because Sullivan et al. show that, even if Slk19 is engineered to be cleaved by another protease, separase is still required for spindle elongation. So, clearly, other aspects of anaphase spindle function require separase.

Spindle elongation in yeast is driven primarily by pushing forces generated from the spindle mid-zone, where microtubules from opposite poles overlap. This pushing force comes from the sliding of antiparallel microtubules by motor proteins as well as from microtubule polymerization. How the integrity of the spindle is maintained while microtubules polymerize and slide past each other is not well understood. However, proteins such as Slk19 and Ase1, which specifically localize to the mid-zone, are obvious candidates to play an important role. To characterize further the role of separase in anaphase spindle movement, Sullivan et al. examined the localization of Slk19 and Ase1 in separase-deficient cells. Strikingly, both proteins were mislocalized: the signal for both proteins was diminished and, rather than being found in the middle of the spindle, both proteins were asymmetrically distributed near one of the spindle 